\title{
A Comprehensive Review of Etiopathogenesis of Oral Submucous Fibrosis
}

\author{
${ }^{1}$ Sukhjinder Kaur Kalra, ${ }^{2}$ Alok A Lathi, ${ }^{3}$ Shilpa A Lathi
}

\begin{abstract}
Accepted as an Indian disease, oral submucous fibrosis, is considered not only a chronic debilitating disease but a potentially malignant condition of oral cavity, strongly associated with the habit of betel nut chewing, that has been sporadically detected in many other western countries and various continents, posing a reason for concern in order to create awareness for such debilitating disease. This article provides an overview of the recent concepts and exact role of areca nut alkaloids and other etiological factors in the pathogenesis of oral submucous fibrosis (OSMF).
\end{abstract}

Keywords: Arecoline, Areca nut, Betel nut, Oral submucous fibrosis.

How to cite this article: Kalra SK, Lathi AA, Lathi SA. A Comprehensive Review of Etiopathogenesis of Oral Submucous Fibrosis. Int J Head Neck Surg 2015;6(2):76-79.

Source of support: Nil

Conflict of interest: None

\section{INTRODUCTION}

Oral submucous fibrosis (OSMF) is a chronic, potentially malignant condition of the oral mucosa which was first described by Schwartz 1952. ${ }^{1}$ Pindborg (1966) defined OSMF as, 'an insidious, chronic disease affecting any part of the oral cavity and sometimes the pharynx. The hallmark of the disease is submucosal fibrosis that affects most parts of the oral cavity, pharynx and upper third of the esophagus leading to dysphagia and progressive trismus due to rigid lips and cheeks. ${ }^{2}$ Although occasionally preceded by and/or associated with vesicle formation, it is always associated with juxtaepithelial inflammatory reaction followed by fibroelastic change of the lamina propria, with epithelial atrophy leading to stiffness of the oral mucosa and causing trismus and inability to eat'. ${ }^{2,3}$

\footnotetext{
${ }^{1-3}$ Senior Lecturer

1,2 Department of Oral Medicine and Radiology, MA Rangoonwala College of Dental Sciences and Research Centre, Pune Maharashtra, India

${ }^{3}$ Department of Prosthodontics, MA Rangoonwala College of Dental Sciences and Research Centre, Pune, Maharashtra, India

Corresponding Author: Sukhjinder Kaur Kalra, Senior Lecturer, Department of Oral Medicine and Radiology, MA Rangoonwala College of Dental Sciences and Research Centre, Pune, Maharashtra, India, Phone: 7774051954, e-mail: drsukhjinderkhosa@gmail.com
}

The main etiological agent causing the disease is confirmed as arecoline in arecanut. ${ }^{4}$ The prevalence of the disease is on the rise in South Asia in the recent years due to the frequently practiced habit of betel chewing. Oral submucous fibrosis is diagnosed on the basis of clinical criteriaincluding oralulceration, paleness of the oral mucosa and burning sensation (particularly in the presence of spicy foods), hardening of the tissue and presence of characteristic fibrous bands. The fibrosis involves the lamina propria and the submucosa and may often extend into the underlying musculature resulting in the deposition of dense fibrous bands giving rise to the limited mouth opening which is a hallmark of this disorder. ${ }^{4}$

Over the past several decades, dental researchers reported different aspects of OSMF. Yet, there is a big lacuna in the present scenario of evidence-based dentistry which correlates the role of critical components of a habit, such as duration, frequency, chewing time to the clinical grading of OSMF.

\section{EPIDEMIOLOGY}

Recent epidemiological data indicate that the number of cases of OSMF has raised rapidly in India from an estimated 2,50,000 cases in 1980 to 2 million cases in 1993. The reasons for the rapid increase of the disease are reported to be due to an upsurge in the popularity of commercially prepared areca nut preparations (pan masala) in India (Ranganathan et al, 2004) and an increased uptake of this habit by young people (Gupta et al, 1998) due to easy access, effective price changes and marketing strategies. Oral submucous fibrosis, now globally accepted as an Indian disease, has one of the highest rates of malignant transformation amongst potentially malignant oral lesions and conditions. ${ }^{4,5}$ The World Health Organization (WHO) definition for an oral precancerous conditiona generalized pathological state of the oral mucosa associated with a significantly increased risk of cancer-accords well with the characteristics of OSMF. It causes significant morbidity (in terms of loss of mouth function as tissues become rigid and mouth opening becomes difficult) and mortality (when transformation into squamous cell carcinoma occurs). ${ }^{6}$

Worldwide, estimates of OSMF show a confinement to Indians and Southeast Asians, with overall prevalence rate in India to be about 0.2 to $0.5 \%$ and prevalence by gender varying from 0.2 to $2.3 \%$ in males and 1.2 to $4.57 \%$ 
in females. ${ }^{5}$ The age range of patients with OSMF is wide ranging between 20 and 40 years of age. ${ }^{7}$ It has been suggested that ingestion of chillies, genetic susceptibility, nutritional deficiencies, altered salivary constituents, autoimmunity and collagen disorders may be involved in the pathogenesis of this condition. ${ }^{3}$ The condition is well recognized for its malignant potential rate of $7.6 \%$ and is particularly associated with use of areca nut in various forms with significant duration and frequency of chewing habits. ${ }^{8,9}$

\section{ETIOLOGY}

Some of the various factors implicated in the etiology of this disease are described below.

\section{Areca and Slaked Lime}

In a habitual betel nut chewer, OSMF may be caused by the amount of tannic acid contained in the betel nut, the influence of mixed calcium powder and the conditional action of arecoline content in betel nut, affecting the vascular supply of oral mucosa and causing neurotropic disorder. This view was further supported by the finding that, addition of slaked lime $\mathrm{Ca}(\mathrm{OH})_{2}$ to areca nut in pan facilitates hydrolysis of arecoline to arecaidine (more potent than arecolin) making this agent available in the oral environment. ${ }^{6}$ The commercially freeze dried products, such as pan masala, gutka and mawa (areca, tobacco and lime) have high concentrates of areca nut per chew and appear to cause OSMF more rapidly than by self-prepared conventional betel quid which contain smaller amounts of areca nut. ${ }^{10,11}$

\section{Chillies}

Consumption of chillies (capsicum annuum and capsicum frutescens) is common in Indians, which is considered as a source of allergen for causing OSMF in a study by Pindborg and Singh. ${ }^{12}$ Capsaicin, which is vanillylamide of 8-methyl-6-nonenic acid, is the active ingredient of chillies, that plays an etiological role in OSMF. ${ }^{3}$

\section{Genetic Predisposition}

Genetic processes have also been thought to be major causative factor. Studies carried out on OSMF indicated that genetic factor allele A6 which is a major histocompatibility complex-class I chain related gene A, confers the risk to develop the disease. Due to involvement of genetic process, raised values of human leukocyte antigen (HLA) A10, B7 and DR3 were found in OSMF patients when compared to normal individuals. ${ }^{13,14}$

\section{Reduced Nutritional Intake}

Higher frequencies of deficiencies of vitamin A, B, C and multiple vitamin deficiencies have been indicated to be of etiologic importance in OSMF. ${ }^{7}$ The deficiency could be precipitated by the effect of defective nutrition due to impaired food intake in advanced cases and may be the effect, rather than the cause of the disease. ${ }^{3}$

\section{Defective Iron Metabolism}

Microcytic hypochromic anemia with high serum iron has also been reported in OSMF. ${ }^{3}$

\section{Immunologic Process}

Natural killer cells also plays vital role in OSMF. Reduced natural killer cell activity was observed in patients with oral leukoplakia and OSMF, so it is suggested that its modulation with interferon may help in control of malignant transformation of oral precancer. Serum immunoglobulin levels of IgA, IgG and IgM are raised significantly in OSMF. ${ }^{15,16}$

\section{Collagen Disorders}

Oral submucous fibrosis is thought to be a localized collagen disease of oral cavity. It is linked to scleroderma, rheumatoid arthritis, duputreyen's contracture and intestinal fibrosis. A link between scleroderma and OSMF has also been suspected on the basis of similarity of histological characteristics. ${ }^{17,18}$

\section{ETIOPATHOGENESIS}

The whole fruit of the areca nut tree can be divided into the unhusked whole fruit, i.e. areca nut and the inner kernel or seed obtained after removing the husk, betel nut. Presence of areca alkaloids impart betel nut its psychotropic and anti helminthic properties. Biochemical studies have identified four kind of alkaloids in areca nut viz, arecoline, arecaidine, guvacine and guvacoline, of which arecoline is the main agent. These alkaloids have powerful parasympathetic properties which produce euphoria and counteract fatigue. Nitrosation of arecoline leads to the formation of areca nut specific nitrosamine namely nitrosoguvacoline, nitrosoguvacine and 3-methyl nitrosominopropionitrile, which alkylate deoxyribonucleic acid, (DNA). Metabolism of these areca nut specific nitrosamine lead to formation of cyanoethyl, which binds with o'methyl guanine in DNA. Prolonged exposure to this irritant leads to malignant transformation (Flow Charts 1 and 2).

Recently suggested pathogenesis of OSMF is by dual action of areca nut. It is suggested that arecoline not only stimulates fibroblastic proliferation and collagen synthesis but also decreases its breakdown. This suggests that arecoline is the active metabolite in fibroblast stimulation (Flow Chart 3). 


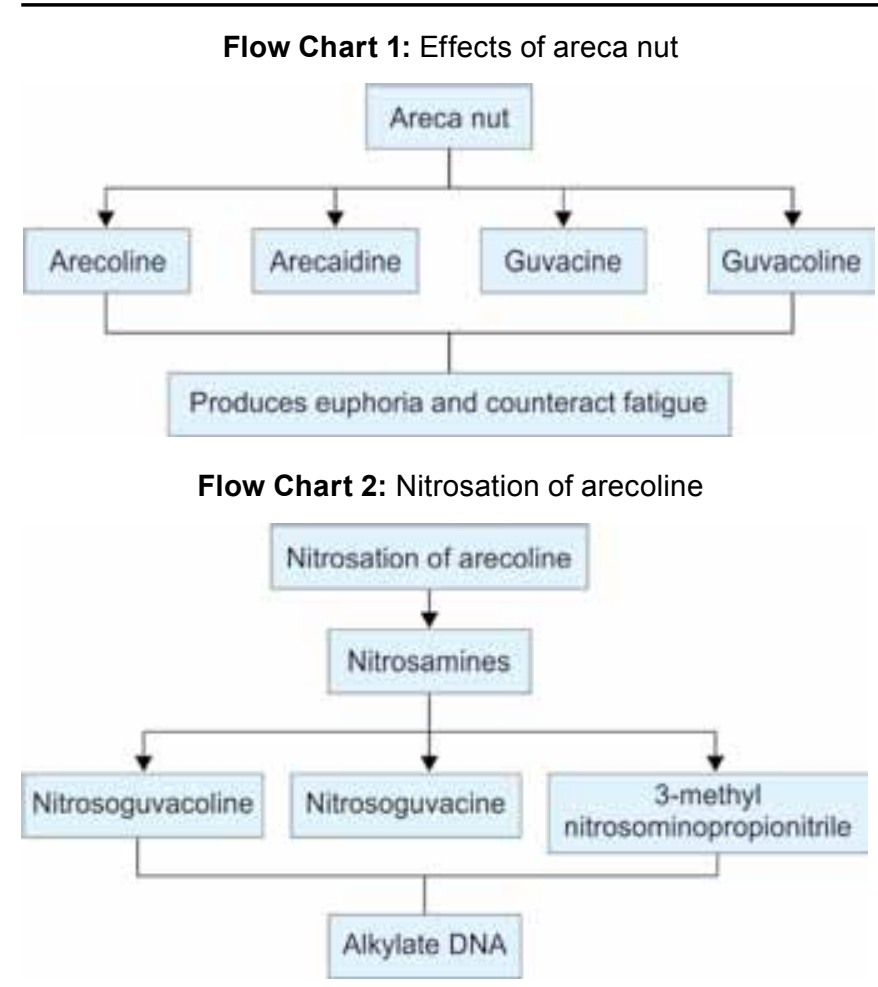

Flow Chart 3: Pathogenesis of oral submucous fibrosis: arecoline

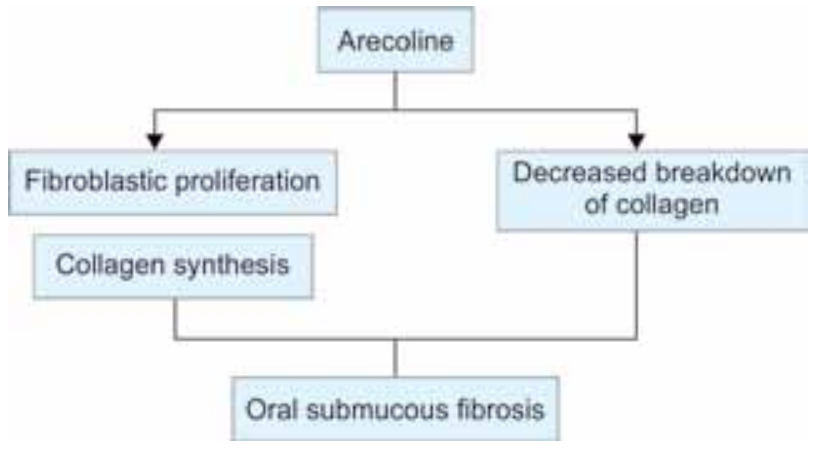

\section{PATHOGENESIS}

\section{Collagen Accumulation}

As described above, OSMF results from increased production of collagen by fibroblasts. In addition to this, there is decreased breakdown leading to accumulation of excessive amount of collagen.

\section{Increased Collagen Production}

Areca nut consumption influences the fibroblasts to differentiate into phenotypes that produce more collagen, due to the presence of the alkaloids, arecadine and arecoline. Arecoline gets converted in to arecadine which is the active metabolite. There is dose dependent increase in production of collagen by fibroblasts under influence of these factors.

Various cytokines are modified is increased in oral submucous fibrosis as follows:

- Increased: Fibrogenic growth factors-transforming growth factor (TGF), platelet derived growth factor (PDGF), basic fibroblast growth factor (BFGF).

- Decreased: Anticollagen effect-interferon
Hence, stimulation of collagen synthesis is manipulated through different mechanisms.

\section{Decreased Collagen Breakdown}

Excessive fibrosis can take place due to reduced degeneration of collagen, by formation of a more stable collagen structure. Tannin, a vital content of betel nut, has the ability to cross-link and thereby stabilizing the collagen. As the disease progresses, type III collagen is almost completely replaced by type I, wherein type I is more resistant to degradation than type III. Another component of betel nut that aids this cross-linking is copper. It is a constituent of enzyme lysyl oxidase. This enzyme also causes cross-linking and makes collagen resistant to degradation by collagenase. Due to action of tannin and copper, collagen that is produced in OSMF is highly resistant to remodeling and phagocytosis. ${ }^{18}$ Fibroblasts aids in remodeling and phagocytosis of collagen. As in OSMF, these fibroblasts are affected and phenotypically changed, they can not degrade collagen. Studies on the effects of arecoline on both normal and OSMF fibroblasts in culture revealed an elevated rate of collagen synthesis by OSMF fibroblasts as compared to normal fibroblasts.

Although the reason for this elevation is not clear, some authors proposed that it could reflect the clonal selection of a highly fibrogenic cell population in the altered tissue under the influence of local factors, such as interleukin-1 from inflammatory cells. This leads to decrease in phagocytosis and accumulation of collagen in oral mucosa. ${ }^{19}$ Glycogen consumption is physiologically related to cellular activity of muscle fibers. Areca nut chewers exhibit over activity of muscles due to repeated, continuous chewing and use of heavy force to crush the hard nut, leading to increased muscle activity resulting in excessive glycogen consumption along with diminished blood supply, following connective tissue changes that leads to muscle degeneration and fibrosis.

\section{Genetic Polymorphisms predisposing to OSMF $^{20,21}$}

Polymorphisms of the genes coding for tumor necrosis factor-alpha (TNF-alpha) has been reported as a significant risk factor for OSMF. Tumor necrosis factor-alpha is known to stimulate fibroblastic proliferation in vitro. Evidences suggest that collagen-related genes are altered due to ingredients in the quid. The genes COL1A2, COL3A1, COL6A1, COL6A3 and COL7A1 have been identified as definite TGF-alpha targets and induced in fibroblasts at early stages of the disease. The transcriptional activation of these procollagen genes by TGF-alpha suggests that it may contribute to increased collagen levels in OSMF. 


\section{OSMF as an Autoimmune Disorder}

Oral submucous fibrosis is also thought to be an autoimmune disease. The presence of various autoantibodies in varying titers is reported in several studies confirming autoimmune basis to the disease. Few studies have reported on HLA typing of OSMF patients and controls. Higher frequencies of OSMF are found in HLA A10, DR3 and DR7 types when compared to an ethnically, regionally and age-matched control group. Although the data on various HLA types, raised autoantibodies and the detection of immune complexes tend to indicate an autoimmune basis for the disease, substantial number of cases and matched controls may be required to verify these finding. $17,22,23$

\section{MANAGEMENT}

Since, the exact cause of OSMF is a matter of conflict, the specific treatment modality can not be achieved. Despite this uncertainity, the frequency of incidence of OSMF can be reduced or eliminated by avoiding the habit of areca nut chewing. If the habit is stopped at early stages, the progression of the disease can be controlled. Following strategies are practiced as current treatment regimens for OSMF: Nutritional support, hyaluronidase, placentral extracts, chymotrypsin, interferon-gamma, immunomodulatory drugs, oral physiotherapy, combined therapy, diathermy, ultrasound, surgical treatment and cryosurgery.

\section{CONCLUSION}

Hence, considering the chronicity of this disease, OSMF is termed as a progressive disorder, that is characterized clinically by stiffening of an otherwise yielding mucosa resulting in difficulty in opening the mouth, worsening the quality of life. Due to the unpredictable etiology, immune response/immune status of individual patient and modality of treatment, no complete success can be guaranteed for OSMF. Owing to the vast literature and various available data, this article has reviewed the various versions of etiopathogenesis and etiologic factors responsible for OSMF, helping to have insight to learn the various roles of those factors in our oral cavity and body system.

\section{REFERENCES}

1. Angadi PV, Rekha K. Oral submucous fibrosis: a clinicopathologic review of 205 cases in Indians. Oral Maxillofac Surg 2011;15(1):15-19.

2. Pindborg J, Sirsat S. Oral submucous fibrosis. Oral Surg Oral Med Oral Pathol 1966;22(6):764.

3. Rajendran R. Oral submucous fibrosis: etiology, pathogenesis and future research. Bulletin of the World Health Organization 1994;72(6):985-996.
4. Ranganathan K, Uma Devi M, Joshua E, Kirankumar K, Saraswathi TR. Oral submucous fibrosis: a case control study in Chennai South India. J Oral Pathol Med 2004;33(5):274-277.

5. Gupta PC, Sinor PN, Bhonsle RB, Pawar VS, Mehta HC. Oral submucous fibrosis in India: a new epidermic. National Med J India 1998;11(3):113-116.

6. Gupta MK, Mhaske Shubhangi, Raghavendra R, Imtiyaz. Oral submucous fibrosis-current concepts in etiopathogenesis. People's journal of scientific research, 2008;6(1):39-44.

7. Haque MF, Harris M, Meghji S, Barrett AW. Immunolocalization of cytokines and growth factors in oral submucous fibrosis. Cytokine, 1998;10(9):713-719.

8. International Agency for Research on Cancer (IARC). Betelquid and areca nut chewing and some areca nut derived nitrosoamines. Lyon IARC, 2004;85(1):123-129.

9. Jacob BJ, Straif K, Thomas G, Ramadas K, Mathew B, Zhang ZF, Rangaswamy S, Hashibe M. Betel quid without tobacco as a risk factor for oral precancers. Oral Oncology 2004;40(7):697-704.

10. Shah N, Sharma PP. Role of chewing and smoking habits in the aetiology of oral submucous fibrosis (OSF): a case control study. J Oral Pathol Med 1998;27(10):475-479.

11. Sinor PN, Gupta PC, Murti PR, Bhonsle RB, Daftary DK, Mehta FS, Pindborg JJ. A case-control study of oral submucous fibrosis with special reference to the aetiologic role of areca nut. J Oral Pathol Med 1990;19(2):94-98.

12. Pindborg JJ. Oral submucous fibrosis: A review. Ann Acad Med Singapore 1989 Sep;18(5):603-607.

13. Shin YN, Liu CJ. Association of CTLA 4 gene polymorphism with oral submucous fibrosis in Taiwan. J Oral Pathol Med 2004;33(4):200-203.

14. Liu CJ. Polymorphism of the MICA gene and risk for oral submucous fibrosis. J Oral Pathol Med 2004;33(1):1-6.

15. Gupta D, Sharma SC. Oral submucous fibrosis: a new treatment regimen. J Oral Maxillofacial Surg 1988;46(10):830-833.

16. Pillai RM, Balaram $P$, Karnan S. Interferon activation of latent natural killer cells and alteration in kinetics of tar- get cell lysis: clinical implications for oral precancerous lesions. Oral Pathol Oral Med Oral Surg Endo 1990;70(4):458-461.

17. Tilakaratne WM, Klinikowski MF, Saku T, Peters TJ, Warnakulasuriya S. Oral submucous fibrosis Review on aetiology and pathogenesis. Oral Oncology, 2005;42(6):561-568.

18. Tsai CC, Ma RH, Shieh TY. Deficiency in collagen and fibronectin phagocytosis by human buccal mucosa fibroblasts in vitro as a possible mechanism for oral submucous fibrosis. J Oral Pathol Med 1999;28(2):59-63.

19. Utsunomiya H, Tilakaratne WM, Oshiro K, Maruyama S, Suzuki M, Ida-Yonemochi H, Cheng J, Sabu T. Extracellular matrix remodeling in oral submucous fibrosis; its stagespecific modes revealed by immunohistochemistry and in situ hybridization. J Oral Pathol Med 2005;34(8):498-507.

20. Vilcek J, Palombella VJ, Henrikson-DeStefano D. Fibroblast growth factor enhancing activity of tumour necrosis factor and its relationship to other polypeptide growth factors. J Experiment Med 1986;163(3):632-643.

21. Rajalalitha P, Vali S. Molecular pathogenesis of oral submucous fibrosis-collagen metabolic disorder. J Oral Pathol Med 2005;34(6):321-328.

22. Canniff JP, Batchelor JR, Dodi K, Harvey W. HLA typing in oral submucous fibrosis. Tissue Antigen 1985;26(2):138-142.

23. Kuo MYP, Chen HM, Hahn LJ, Hsieh CC, Chiang CP. Collagen biosynthesis in human OSF fibroblast cultures. J Dent Res 1995;74(11):1783-1788. 